\title{
Correction: MobiDetails: online DNA variants interpretation
}

\author{
David Baux (1) Charles Van Goethem - Olivier Ardouin - Thomas Guignard (1) - Anne Bergougnoux • \\ Michel Koenig · Anne-Françoise Roux
}

Published online: 10 December 2020

(c) European Society of Human Genetics 2020

Correction to: European Journal of Human Genetics https://doi.org/10.1038/s41431-020-00755-z

Table 2 of the original article contained some mistakes. The corrected table has been updated in the original article and is also included below, with the updated values denoted with three asterisks: $* * *$.

In addition, the "Comparison with other online platforms" paragraph has also been slightly modified in the original article according to these changes:
"However, VEP and MARRVEL2 lack several useful features such as automated literature search. MARRVEL2 and VarSome do not provide splice site predictions" has become:

"MARRVEL2 lack several useful features such as automated literature search. MARRVEL2 and VarSome do not provide extensive splice site predictions".

Table 2 Comparison of features between MobiDetails and similar software.

\begin{tabular}{|c|c|c|c|c|c|}
\hline & & MobiDetails & VarSome & VEP web & MARRVEL \\
\hline \multirow{6}{*}{$\begin{array}{l}\text { Access and technical } \\
\text { aspects }\end{array}$} & Free usage for academics & $\mathrm{y}$ & $\mathrm{y}$-limited & $\mathrm{y}$ & $\mathrm{y}$ \\
\hline & Free API usage for academics & $\mathrm{y}$ & $\mathrm{n}$ & $\mathrm{y}$ & $\mathrm{y}$ \\
\hline & Local installation & $y^{*}$ & $\mathrm{n}$ & $\mathrm{y}$ & $\mathrm{n}$ \\
\hline & User friendly & $\mathrm{y}$ & $\mathrm{y}$ & $\begin{array}{l}\mathrm{n} \text { - but extensive } \\
\text { options }\end{array}$ & $\mathrm{y}$ \\
\hline & Encrypted connection by default*** (https) & $\mathrm{y}$ & $\mathrm{y}$ & $\mathbf{n}^{* * * *}$ & $\mathrm{n}$ \\
\hline & Responsive website & y & $\mathrm{y}$-limited & $\mathrm{n}$ & $\mathrm{y}$ \\
\hline \multirow[t]{10}{*}{$\begin{array}{l}\text { Specific content for } \\
\text { interpretation }\end{array}$} & Custom annotations & $\mathrm{y}$-registered users & $\mathrm{y}$-registered users & $\begin{array}{l}\mathrm{y} \text {-only on local } \\
\text { instances }\end{array}$ & $\mathrm{n}$ \\
\hline & $\begin{array}{l}\text { Selectable wild-type and mutant } * * * \text { DNA } \\
\text { sequence surrounding variants }\end{array}$ & $\mathrm{y}$ & $\mathrm{n}$ & $\mathrm{n}$ & $\mathrm{n}$ \\
\hline & $\begin{array}{l}\text { gnomADv3: whole genome population } \\
\text { variant frequencies }\end{array}$ & $\mathrm{y}$ & $y^{* * *}$ & $\mathrm{n}$ & $\mathrm{n}$ \\
\hline & Extensive*** splice site predictions & $\mathrm{y}$ & $\mathrm{n}$ & $\mathrm{y}$ & $\mathrm{n}$ \\
\hline & miRNA target sites predictions & $\mathrm{y}$ & $\mathrm{n}$ & $\mathrm{n}$ & $\mathrm{n}$ \\
\hline & Literature search & $\mathrm{y}$-LitVar & $\mathrm{y}$-MasterMind & $\mathbf{y}^{* * *}$ & $\mathrm{n}$ \\
\hline & LOVD occurrences & $\mathrm{y}$ & $\mathrm{n}$ & $\mathrm{n}$ & $\mathrm{n}$ \\
\hline & Pdf export & $\mathrm{y}$ & $\mathrm{n}$ & $\mathrm{n}$ & $\mathrm{n}$ \\
\hline & hg38 coordinates & $\mathrm{y}$ & $\mathrm{y}$ & $\mathrm{y}$ & $\mathrm{n}$ \\
\hline & All small variants type** & $\mathrm{y}$ & $\mathrm{y}$ & $\mathrm{y}$ & $\begin{array}{l}\mathrm{n} \text {-only } \\
\text { substitutions }\end{array}$ \\
\hline
\end{tabular}

*Requires the SQL scheme available upon request.

**Substitutions and small insertions/deletions.

***Updated values. 